\title{
Knowing when to stop: Rhythms of locomotor activity in the high-shore limpet, Cellana grata Gould
}

\author{
David R. Gray ${ }^{a}$, Gray A. Williams ${ }^{\mathrm{b}, *, 1}$ \\ a NAFC Marine Centre, Port Arthur, Scalloway, Shetland Islands, UK \\ b The Swire Institute of Marine Sciences and the Division of Ecology \& Biodiversity, The School of Biological Sciences, The University of Hong Kong, Pokfulam Road, Hong Kong
}

\section{A R T I C L E I N F O}

Article history:

Received 9 March 2010

Received in revised form 17 June 2010

Accepted 17 June 2010

Available online $\mathrm{xxxx}$

\section{Keywords:}

Behaviour

Circatidal

Endogenous rhythms

Tropical rocky shore

\begin{abstract}
A B S T R A C T
The high shore limpet, Cellana grata, forages whilst awash, moving upshore with the rising tide and retreating downshore on the ebbing tide to become inactive in refuges. Spraying inactive, emersed individuals with seawater at low tide invokes a locomotory response, with limpets moving up the shore. Controlled laboratory experiments under continuous white or red light (to simulate light or dark periods respectively) and continuous emersion, immersion or seawater spray showed that $C$. grata possesses a freerunning endogenous rhythm of locomotor activity. This rhythm was maintained over 30 days in continuous seawater spray and white light. Maximum entropy spectral analysis (MESA) revealed two major components to this rhythm, at $7.2 \mathrm{~h}$ and $12.4 \mathrm{~h}$. The $12.4 \mathrm{~h}$ component is of a circatidal nature and appears to initiate activity, allowing individuals to anticipate immersion by the incoming tide, although this clock can be overridden by strong wave splash or spraying vigorously with seawater. The $7.2 \mathrm{~h}$ period, however, was the most significant component and is suggested to act as a stopwatch enabling the limpet to assess the duration of each foraging excursion in order to prevent being stranded at the wrong height on the shore. The environmental stimulus for both components of the endogenous rhythm in C. grata appears to be the time of first exposure to wave wash from the incoming tide. C. grata, therefore, has behavioural rhythms entrained to initiate and also terminate activity, which play a role in the limpet maintaining a fixed vertical level on the shore when inactive.
\end{abstract}

(c) 2010 Published by Elsevier B.V.

\section{Introduction}

Many intertidal animals exhibit rhythmic behavioural patterns, synchronising their activity with external environmental variables such as tidal cycles, day-night cycles, monthly spring-neap cycles and even annual seasonal changes (reviewed by Hawkins \& Hartnoll, 1983; Naylor, 1988; Little, 1989; Morgan, 2007). Many of these patterns are primarily driven by changes in external conditions, whilst some species continue to exhibit free-running activity rhythms under constant conditions. The circatidal and circadian rhythmicity of the behaviour and physiology of many mobile intertidal animals is well documented (e.g. Naylor, 1958; Zann, 1973) and the temporal synchronization of foraging behaviour with cyclical environmental variables has been suggested to ensure optimal resource utilization and the avoidance of stressful situations (Naylor, 1988, 1989; Williams and Little, 2007).

Slow-moving, intertidal herbivorous gastropods, such as limpets, exhibit rhythmic foraging excursions away from their home scars or

\footnotetext{
* Corresponding author. Tel.: + 852 28092179; fax: +852 28092197.

E-mail address: hrsbwga@hkucc.hku.hk (G.A. Williams).

${ }^{1}$ Authorship is alphabetical.
}

refuge areas, to which they often return upon completion of their feeding cycle (Orton, 1929; Branch, 1981; Little, 1989; Gray and Hodgson, 1997). Apart from some equivocal experiments (Funke, 1968) only a few studies have indicated that the on-shore rhythmic foraging activity of limpets is endogenously controlled (Della Santina and Naylor, 1993; Gray and Hodgson, 1999). Clear endogenous rhythms have only been reported in a few species of intertidal molluscs including bivalves (Beentjes and Williams, 1986; Akumfi and Naylor, 1987), gastropods (Zann, 1973, Petpiroon and Morgan, 1983) and chitons ( $\mathrm{Ng}$ and Williams, 2006), possibly due to the difficulties involved with maintaining these animals under laboratory conditions.

Cellana grata Gould is abundant in the mid-high shore of moderately exposed to exposed rocky shores in Hong Kong (Morton and Morton, 1983; Williams, 1993), and forages whilst awash, moving up shore with the rising tide and downshore on the ebb, rarely being completely submerged (Williams and Morritt, 1995; Davies et al., 2006). This limpet appears to be an opportunistic forager, as individuals are active during periods of high wave action, when they are splashed by seawater, even at the time of predicted low water (e.g., during typhoons, Williams and Morritt, 1995). Individuals do not, however, follow the tide to its lowest point but take refuge in habitats that offer shelter from high temperatures at $\sim 1.75-2.0 \mathrm{~m}$ 
above Chart Datum (C.D.). If C. grata fails to retreat to a suitable refuge after foraging, especially in the transition period between the cool/dry and hot/wet monsoon (see Kaehler and Williams, 1996 for description of Hong Kong climate) when it migrates lower down the shore, animals can suffer thermal and desiccation stress and subsequent mortality (Williams, 1994; Williams and Morritt, 1995; Ngan, 2006). The maintenance of a rhythmic locomotry behaviour and subsequent habitat selection would, therefore, appear to have a selective function for this limpet. The present study, therefore, aimed to establish whether the rhythms of locomotor activity exhibited by $C$. grata on the shore have an endogenous component and, if so, what those components may be.

\section{Materials and methods}

\subsection{On-shore observations and collections}

All experiments were conducted at The Swire Institute of Marine Science, Hong Kong $\left(22^{\circ} 17^{\prime} \mathrm{N}, 114^{\circ} 09^{\prime} \mathrm{E}\right)$ and on the shores within the Cape d'Aguilar Marine Reserve. Hong Kong tides can be broadly described as mixed semi-diurnal, with tidal range $\sim 2.5 \mathrm{~m}$ (see Williams, 1994 and Morton et al., 1996) and spring tides of unequal amplitude. The normal resting height of $C$. grata on the shores around Cape d'Aguilar is $\sim 1.75-2.0 \mathrm{~m}$ above C.D.

To determine whether wave action stimulates locomotor activity in C. grata, limpets were sprayed with seawater using hoses. During low tide, when animals were inactive, small groups of limpets were sprayed via hosepipes with unfiltered seawater from a large holding tank. Due to storage and transfer time, the seawater sprayed had a temperature $\sim 1.5^{\circ} \mathrm{C}$ higher than ambient seawater temperatures $\left(28-29.5^{\circ} \mathrm{C}\right)$.

On two separate day-time low tides in July 1994, groups of limpets were sprayed and not sprayed (as a control) at different sites (Cape Wall and Cape Slope, see Williams and Morritt, 1995). Four groups of limpets were monitored on 07/07/1994 (90 min prior to low tide, two groups of 14 limpets sprayed and two groups of 9 and 14 not sprayed) and 18/07/1994 (at the time of low tide, two groups of 18 and 29 sprayed animals; and two groups of 30 individuals not sprayed). Animals were scored at $\sim 20-30$ min intervals as either inactive (inert, firmly attached to the rock surface within a $15 \mathrm{~s}$ observation period); active, but no movement (lifting off the rock but stationary, pallial tentacles visible) or moving (movement seen within 15 s). On 18/07/ 1994, the height relative to C.D. of the animals was also recorded at the Cape Wall site, as this site is a vertical wall where tidal heights can be readily estimated (Williams and Morritt, 1995).

\subsection{Laboratory experiments to test for endogenous rhythms}

To determine whether locomotor activity in Cellana grata is endogenously controlled, limpets were collected from a $150 \mathrm{~m}$ stretch of south-facing shore line at Cape d'Aguilar for laboratory experiments in September-October 1997. Easily removable animals (28.5$37 \mathrm{~mm}$, from the same cohort) were collected by hand whilst active and awash by the tide. Once collected, the limpets were immediately returned to the laboratory (a distance of approximately $400 \mathrm{~m}$ ), placed in 40-l experimental tanks and allowed to attach to one side of the tank. This side of the tank was fenced to prevent limpets moving off this surface and, therefore, out of the field of view. The animals were then immediately transferred to an experimental area ( $3 \mathrm{~m} \times 2 \mathrm{~m} \times 2 \mathrm{~m}$ ), which was separated from the main aquarium with blackout curtains and monitored using a video camera (Sony, CCD - TR3E) connected to a time lapse video recorder (Panasonic, AG - 6730) outside the aquarium. In this way the limpets could be observed without any disturbance. Within the experimental area, red or white light could be used and photoperiod varied whereas temperature and relative humidity were constant. At the end of the experiments limpets were returned to an area of the shore from which further collections were not made.

Ten limpets were placed in each of three 40-1 experimental tanks $(\Sigma \mathrm{n}=3 \times 10=30$; data was not pooled) and different treatments applied to each tank to simulate three stages of the tidal cycle. All treatments experienced relatively constant temperature (25.5$27.5^{\circ} \mathrm{C}$ ) and relative humidity (>90\%). Limpets were kept either permanently immersed; constantly emersed or constantly sprayed by seawater (to simulate awash conditions). The experiment was repeated once in red light ( $80 \mathrm{~W}$ Phillips Par 38 Flood) for a period of $96 \mathrm{~h}$ and once in white light (120 W Phillips Par 38 Flood) for $48 \mathrm{~h}$. Movement of Cellana grata is not inhibited by red light (Williams and Morritt, 1995) as is also the case for Patella vulgata (Little and Stirling, 1985) and Helcion pectunculus (Gray, 1997). The red light was, therefore, an attempt to simulate darkness for the limpets and yet be able to maintain observations. Many limpets were dead at the end of the red light experiment in both the emersed and immersed tanks, therefore, the duration of the white light experiment was reduced to $48 \mathrm{~h}$ to minimize mortality. In order to explore potential variations in any rhythms observed over the full spring-neap tidal cycle, 20 Cellana grata were maintained for 30 days (i.e., two full spring-neap cycles) in a 150-l experimental tank under constant white light and seawater spray.

Video tapes were viewed on fast-forward and the observations divided into 15 time-lapsed minute intervals or "bins". To standardize scoring, the same person scored all the tapes. The number of limpets active within the 15-minute bin was recorded along with the number of limpets visible. If a limpet moved off the side of the tank, it was excluded from records until it returned. Activity was defined as turning movements as well as actual displacement and calculated as the percentage of limpets active of the individuals visible. To determine the presence of rhythms, Maximum Entropy Spectral Analysis (MESA; Dowse and Ringo, 1989) employed in conjunction with autocorrelation (e.g., Statgraphics 1991, as advised by Palmer, 1995) were used on the month-long observations.

\section{Results}

\subsection{On-shore observations}

In both experiments on the shore, non-sprayed limpets did not show signs of being active in any of the control groups, except at the end of the experiment on 18/07/1994 when waves from the incoming tide reached the lowest animals, which then started to move. Limpets sprayed with seawater to simulate wave action, however, became active within $30 \mathrm{~min}$ of the initiation of spraying (Fig. 1). Limpets initially raised their shells off the substratum, extended their pallial tentacles, and then started moving. Within $1 \mathrm{~h}$, between 60 and $80 \%$ of the limpets in both groups were moving (Fig. 1). In general, animals were stimulated to move by strong jets of water. On 07/07/1994 spray was terminated after $1 \mathrm{~h}$, after which most animals stopped moving, and all became inactive within $1 \mathrm{~h}$ as the rock surface started to dry (Fig. 1a).

On 18/07/1994 animals were sprayed continuously for $2.5 \mathrm{~h}$ (after which waves from the incoming tide wetted the experimental population). Sprayed limpets, as in the previous experiment, rapidly became active and within $30 \mathrm{~min},>60 \%$ of the two groups were moving, and continued to move when the animals were washed by the incoming tide (Fig. 1b). Sprayed limpets moved up-shore by $\sim 40 \mathrm{~cm}$ from their resting tidal height after $2 \mathrm{~h}$ (Fig. 2).

\subsection{Laboratory experiments to test for endogenous rhythms}

In the laboratory, activity of Cellana grata was found to be rhythmical throughout both the red and white light experiments, under all experimental conditions (Fig. 3). Most limpets were active 
07/07/1994

(a) Cape Wall

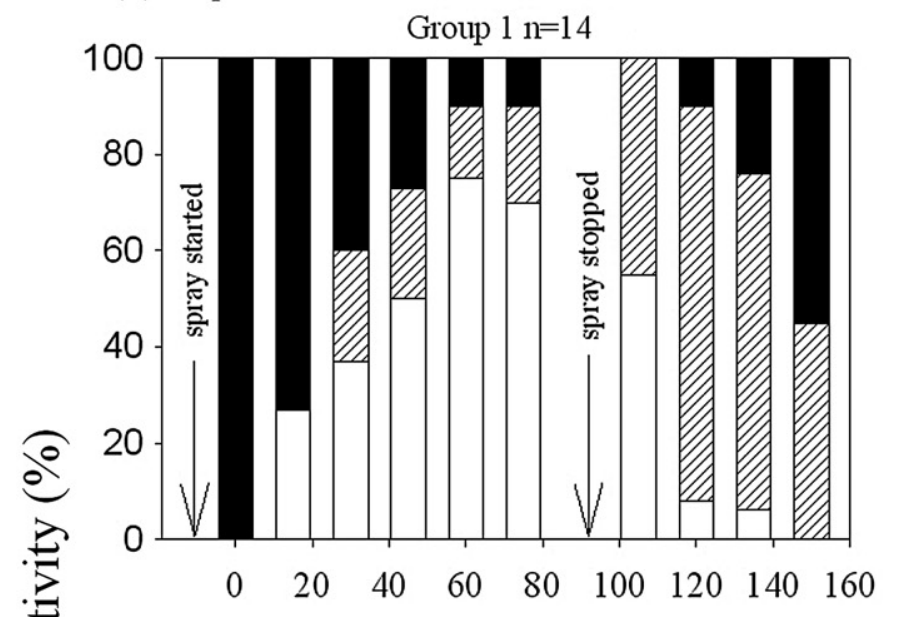

Cape Slope

苋 1

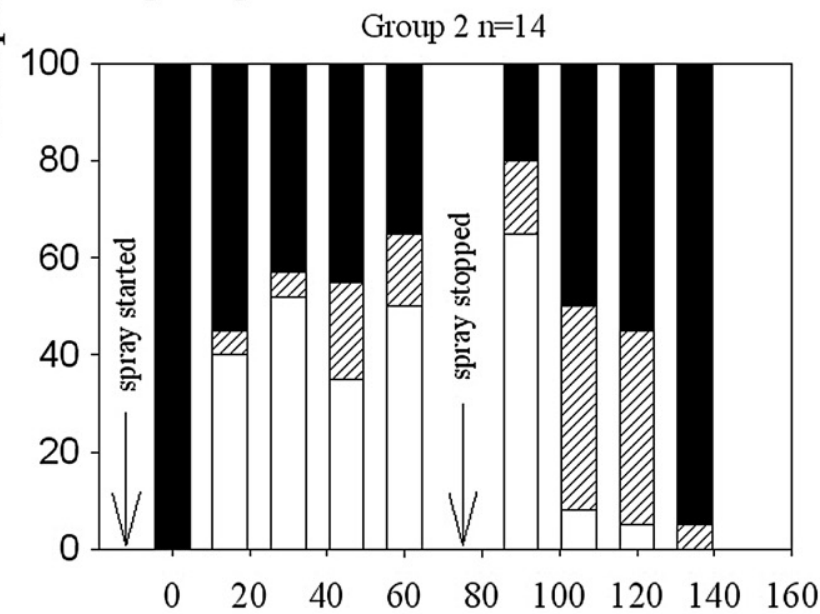

(b) Cape Wall

$18 / 07 / 1994$

\section{Group $1 \mathrm{n}=18$}

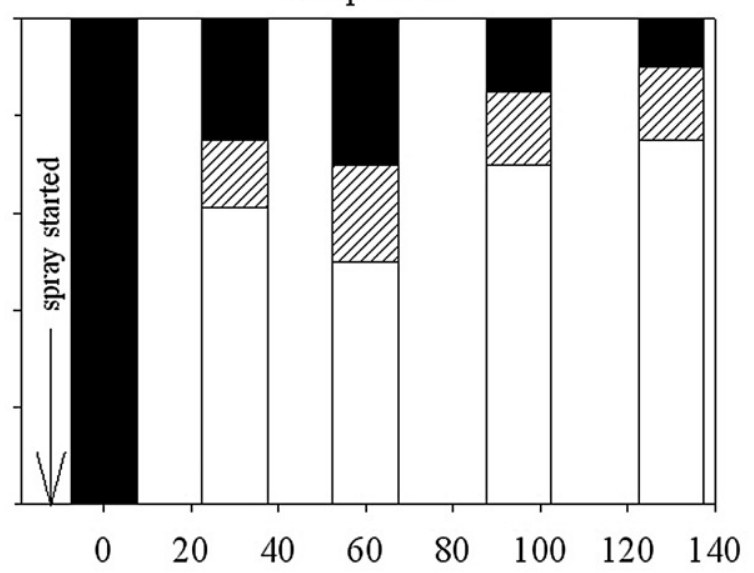

\section{Cape Slope}

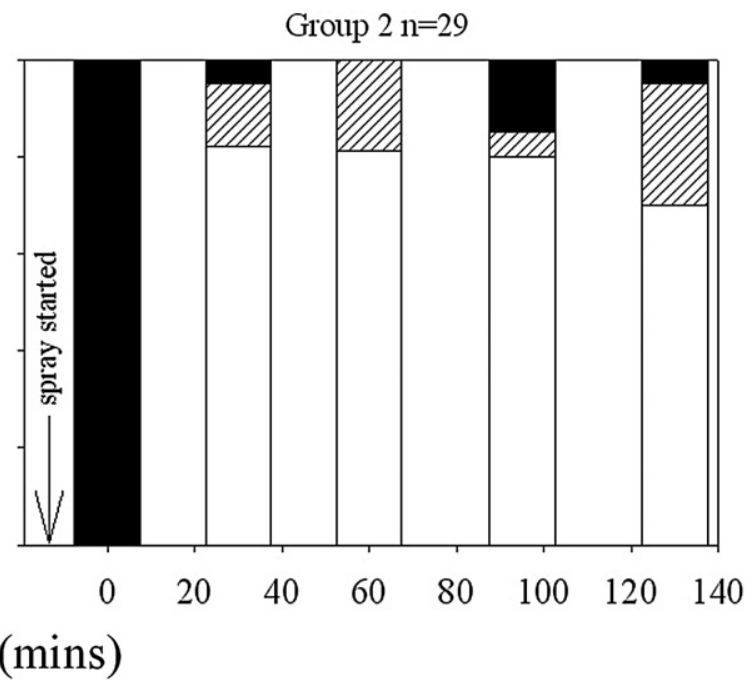

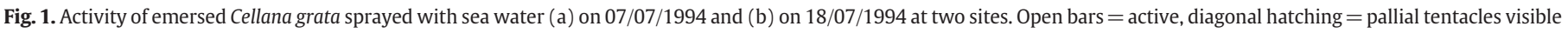
and filled bars = inactive.

during times of the expected incoming tide and remained active for periods of up to $6 \mathrm{~h}$. The termination of activity appeared to be synchronised with the time when the tidal level on the shore would have fallen below the normal resting height of $C$. grata $(\sim 1.75 \mathrm{~m}$ above C.D., Fig. 3). The rhythmic nature of the locomotor behaviour in this limpet, therefore, indicates the presence of an internal biological clock.

The intensity of activity also showed a rhythmic component. Activity was greater during the period of the higher of the high tides periods ( $\sim 60-70 \%$ activity) as compared to the lower, high water period on the shore ( $30-40 \%$ activity, Fig. 3 ) under both white and red light conditions. This pattern was maintained for the first two days in the red light treatments (Fig. 3) after which activity patterns became less clear, especially in the immersed and emersed treatments where activity intensity decreased and some animals died. In contrast, activity intensity remained fairly constant in the spray treatment, suggesting this method was more appropriate for maintaining limpet populations in the laboratory. Experiments conducted in white light confirmed this pattern, with the animals in the spray tank maintaining a strong rhythm for four tidal cycles, with much weaker, but similarly timed, peaks and some mortality in the immersion and emersion treatments (Fig. 3). Animals under continual immersion immediately moved up the side of the tank towards the water-air interface at the start of both experiments. The wire fence prevented the animals becoming emersed and they stopped moving but remained at the top of the tank. Within $24 \mathrm{~h}, 30 \%$ of the immersed limpets were dead and after $96 \mathrm{~h} 70 \%$ mortality had been observed in the red light experiment. In the white light experiment 60\% mortality of animals was recorded after $48 \mathrm{~h}$.

The percentages of limpets that were active every hour throughout 30 days observation in constant conditions of white light and seawater spray spanned 0-95\% and activity appeared rhythmic throughout the experiment (Fig. 4). The majority of limpets were active during times of expected rising and falling tides i.e., two activity bouts per high tide. Intensity of activity peaks did not differ between day and night tidal cycles suggesting the lack of a circadian component. Activity did decrease during the course of the experiment, from peaks of almost $100 \%$ activity during the first 4-5 days to $\sim 50 \%$ activity for the remaining period of the experiment. MESA analysis confirmed the presence of two major activity peaks, one at $7.2 \mathrm{~h}$ and one at $12.4 \mathrm{~h}$ (Fig. 5a), and autocorrelation verified the significance of these peaks by identifying those peaks that were 


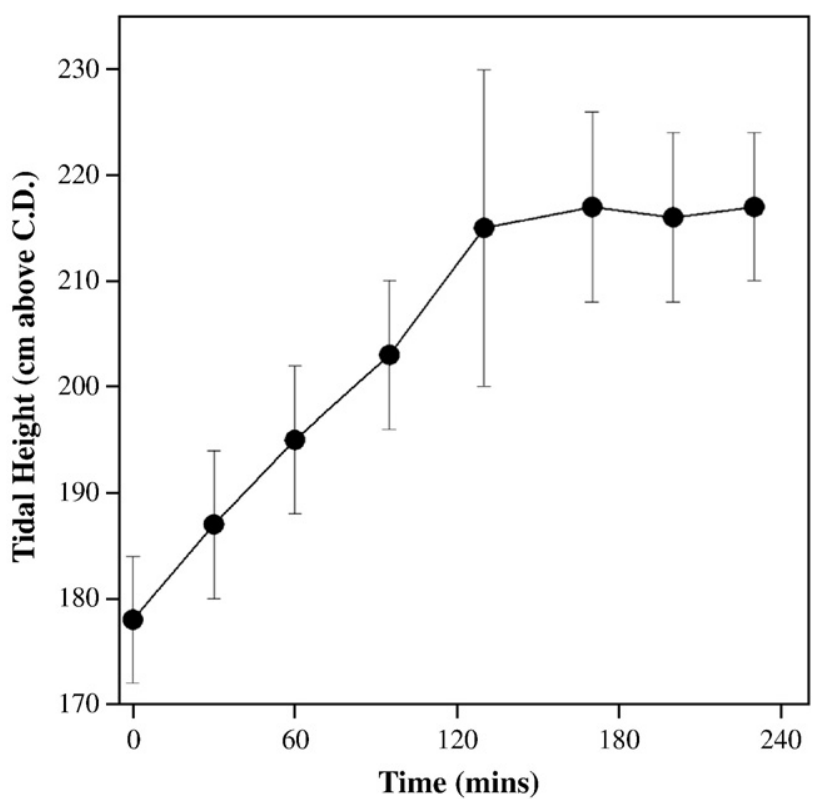

Fig. 2. Mean height of Cellana grata ( $\mathrm{cm}$ above C.D., \pm S.D., $\mathrm{n}=18$ ) from time of initial spraying with seawater on 18/07/1994.

statistically different $(\mathrm{p}<0.05)$ from the inherent background activity (Fig. 5b). A further peak was present on the MESA output at $24.8 \mathrm{~h}$, which is a multiple of the circatidal component (see significant peaks in autocorrelation). It was not possible to test for the presence of a circasemilunar component as the 30-day experimental period only included two spring-neap cycles and so a statistical comparison was not practical.

\section{Discussion}

Cellana grata showed a clear endogenous pattern of locomotor activity, initiating activity prior to the high tide and terminating activity after $\sim 7 \mathrm{~h}$, on the ebbing tide. This closely matches patterns observed on the shore, where $C$. grata becomes active when washed by waves and moves up shore with the flooding tide. As the tide recedes, $C$. grata moves down shore and terminates activity at a fixed tidal height, above the low water level, becoming inactive in suitable refuges (see Williams and Morritt, 1995 and Davies et al., 2006 for description of behaviour on the shore). These results are in contrast to those of Liu (1993) who recorded no endogenous rhythm for C. grata or Cellana toreuma, although this is likely a result of inadequacies of the tidal tank and the holding periods used (the tank was very shallow and had no "awash" phase, Liu, 1993). Evidence for endogenous rhythms in limpets is sparse, but Patella vulgata (Della Santina and Naylor, 1993) and Helcion pectunculus (Gray and Hodgson, 1999) exhibit similar endogenous activity patterns, being active at low tide. Gray and Hodgson (1999) suggested that the exogenous entrainment factor in both cases is the time of exposure to air, although light above a threshold intensity does inhibit locomotor activity in $H$. pectunculus. In both P. vulgata (Della Santina and Naylor, 1993) and H. pectunculus the rhythm of locomotor activity was suppressed after a prolonged period of exposure (approx. $24 \mathrm{~h}$ ), which does not appear to be the case for $C$. grata.

Two clock systems appear to be involved in controlling locomotor activity in Cellana grata. The first and most significant has a period of
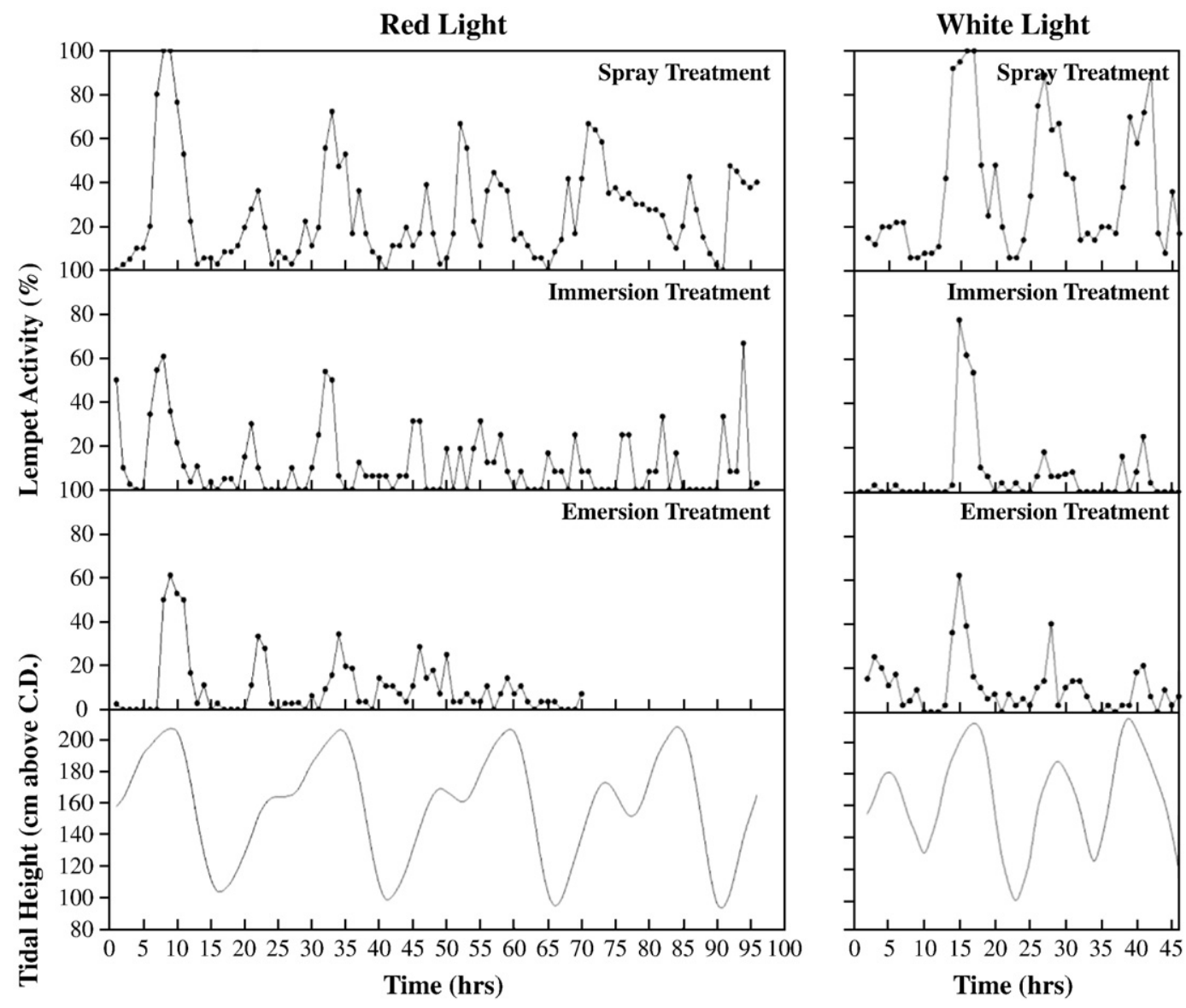

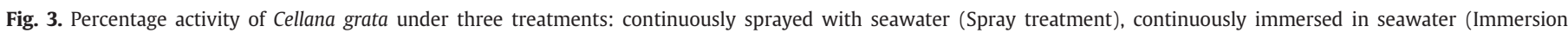

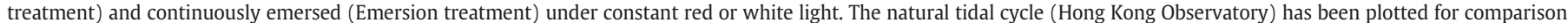



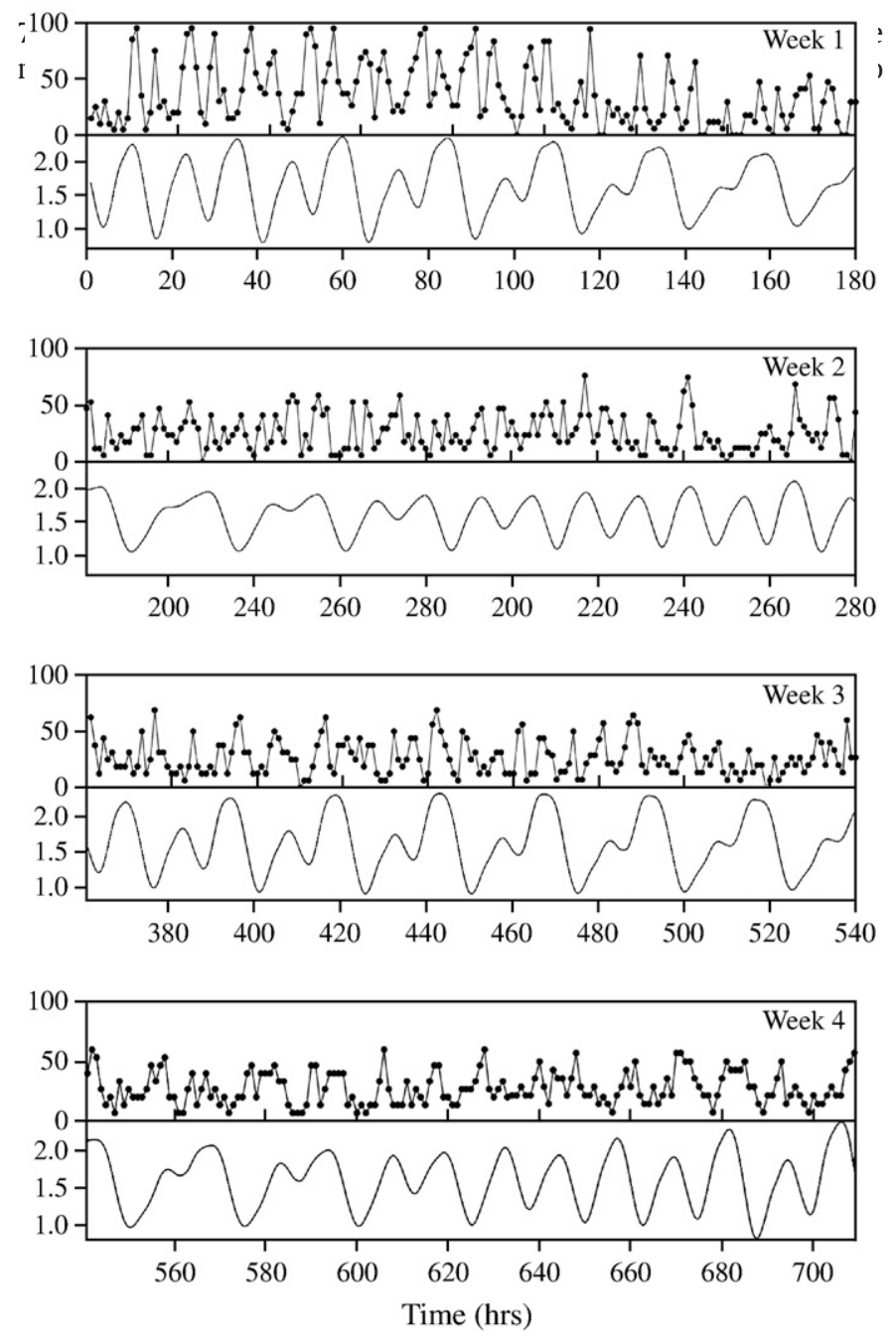

Fig. 4. Percentage activity of Cellana grata under conditions of constant white light and seawater spray for 30 days. The natural tidal cycle (Hong Kong Observatory) is plotted for comparison.

the time when the ebbing tide again reaches the same tidal height (see Fig. 4, Williams and Morritt, 1995). This allows the limpets a 'stopwatch' capability i.e., they are able to gauge when to stop movement. C. grata, therefore, do not follow the ebb tide down to low water but stop at a certain, presumably optimal, height on the shore as determined by their stopwatch clock.

The second significant clock system has a period of $12.4 \mathrm{~h}$ and is circatidal in nature. This gives the animal information about when to expect an incoming tide. Cellana grata will, however, start to move when sprayed with seawater or washed by waves, irrespective of the state of the tide. This may be a safety mechanism to allow for the somewhat unpredictable nature of the tidal regime in Hong Kong due to both the mixed semidiurnal tides, and also typhoon season/high wave action which can effectively override the tidal cycle on local shores which have a narrow tidal range. Movement can be initiated by wave action and artificial spraying with seawater (simulating an incoming tide) with animals moving up shore in a manner similar to their natural behaviour on an incoming tide. This indicates that the endogenous clock can be over-ridden by such stimuli, further supporting the theory that $C$. grata is an opportunistic forager, feeding whenever conditions are suitable (Williams and Morritt, 1995; Davies et al., 2006; Ngan, 2006).

Endogenous locomotor activity patterns may, in some circumstances, play a role in avoidance of unfavourable environmental conditions (Little and Stirling, 1985; Naylor, 1988). The endogenous
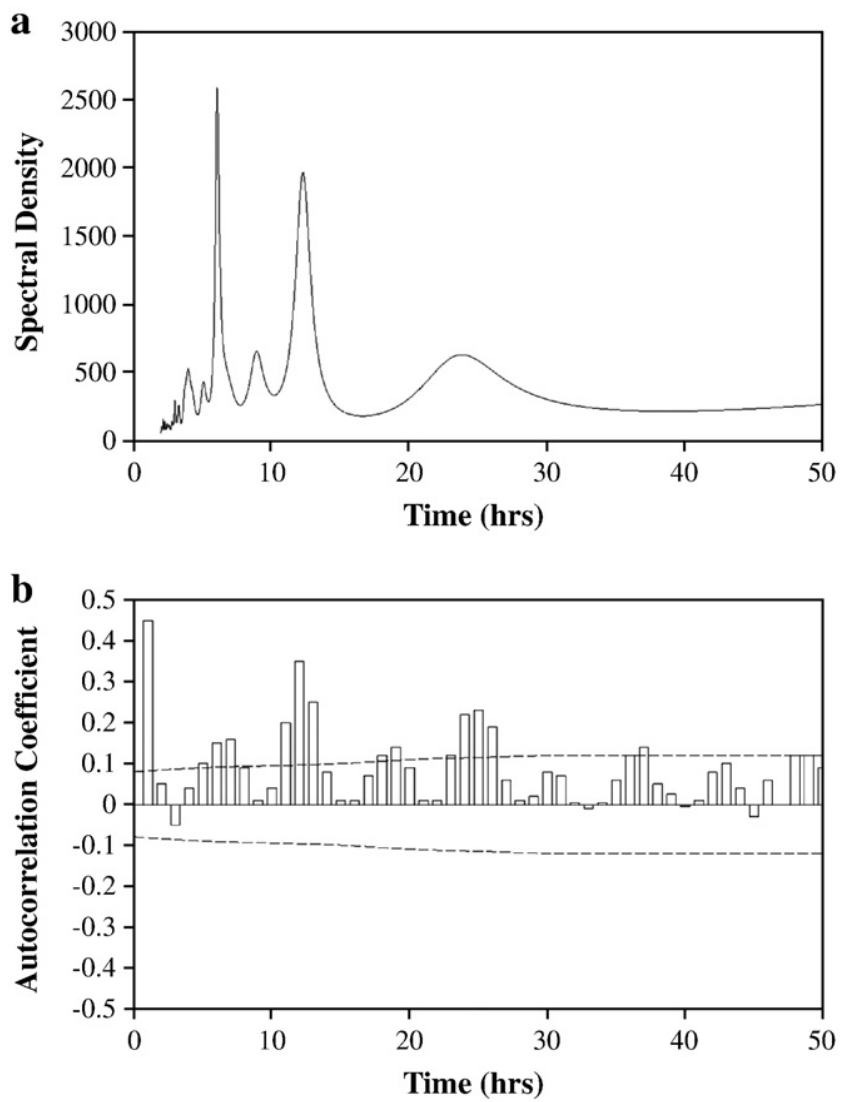

Fig. 5. (a) MESA analysis to detect peaks in activity data obtained from Cellana grata maintained under constant conditions of white light, seawater spray, temperature and relative humidity for 30 days. (b) Autocorrelation analysis, dotted lines indicate $95 \%$ confidence intervals.

rhythm exhibited by Cellana grata seems to support this hypothesis. The circatidal component of the rhythm ensures that the individual is active as the tide rises and falls and also gives the limpet the ability to predict the time available for foraging before moving back down the shore. This prevents the animal from being immersed by the incoming tide and ensures re-location of its resting height on the shore. Observations during the present study suggest that $C$. grata is not able to survive prolonged periods of submergence, and avoids immersion.

Cellana grata does not appear to have a circadian component to its locomotory rhythm, although this is exhibited by both $P$. vulgata (Della Santina and Naylor, 1993) and H. pectunculus (Gray and Hodgson, 1999), and by the chiton, Acanthopleura japonica on Hong Kong shores ( $\mathrm{Ng}$ and Williams, 2006). If the primary aim of the rhythm is to prevent the animals from being submerged for a prolonged period of time then the main component of the rhythm would be synchronised with the tidal cycle and not with the daynight cycle. The fact that light does not inhibit foraging activity in $C$. grata also points towards the day-night cycle being of little importance in the biological clock. If the function of the rhythm is to avoid immersion then the logical zeitgeber to the rhythm is likely to be associated with the tidal cycle, possibly the incoming tide. The experiments and periodicity of the rhythms described here match the observed patterns of foraging behaviour for $C$. grata during spring tide cycles in Hong Kong. Hong Kong, however, experiences mixed semidiurnal tides, especially during summer when neap tides are almost diurnal. Further experimental work is clearly required to verify whether a circasemilunar component is present which would give the animal an added advantage by being able to predict the mixed semidiurnal tides experienced on Hong Kong shores. 


\section{Acknowledgements}

We are grateful to The Laurence Caplin Scholarship in Marine Biology, administered by the Committee of Management of The Swire Institute for Marine Science (HKU), which provided funds for accommodation and travel to DRG. Thanks to colleagues of the Hard Rock Ecology Group (HKU) who commented on the manuscript, Dr D. Dowse who provided the MESA package, Ms Kelly Lau for help with fieldwork and Ms Cecily Law who prepared the figures. Equipment was funded by a CRCG (HKU) grant to GAW. We are grateful for the helpful comments on the MS from Prof George M Branch. [RH]

\section{References}

Akumfi, C.A., Naylor, E., 1987. Temporal patterns of shell-gape in Mytilus edulis. Mar. Biol. 95, 237-242.

Beentjes, M.P., Williams, B.G., 1986. Endogenous circatidal rhythmicity in the New Zealand cockle Chione stutchburyi (Bivalvia, Veneridae). Mar. Behav. Phys. 12, 171-180.

Branch, G.M., 1981. The biology of limpets: physical factors, energy flow and ecological interactions. Oceanogr. Mar. Biol. Ann. Rev. 19, 235-380.

Davies, M.S., Edwards, M., Williams, G.A., 2006. Movement patterns of the limpet Cellana grata (Gould) observed over a continuous period through a changing tidal regime. Mar. Biol. 149, 775-787.

Della Santina, P., Naylor, E., 1993. Endogenous rhythms in the homing behaviour of the limpet Patella vulgata Linnaeus. J. Moll. Stud. 59, 87-91.

Dowse, H.B., Ringo, J.M., 1989. The search for hidden periodicities in biological time series revisited. J. Theo. Biol. 148, 139-144.

Funke, W., 1968. Heimfindevermögen und Ortstreue bei Patella L. (Gastropoda, Prosobranchia). Oecologia 2, 19-142.

Gray, D.R., 1997. Studies of the biology and ecology of the high score South African limpet, Helcion pectunculus (Mollusca: Patellogastropoda). PhD Thesis, Rhodes University, South Africa.

Gray, D.R., Hodgson, A.N., 1997. Temporal variation in foraging behaviour of Patella granularis (Patellogastropoda) and Siphonaria concinna (Basommatophora) on a South African shore. J. Moll. Stud. 63, 121-130.

Gray, D.R., Hodgson, A.N., 1999. Endogenous rhythms of locomotor activity in the highshore limpet Helcion pectunculus (Patellogastropoda). An. Behav. 57, 387-391.

Hawkins, S.J., Hartnoll, R.G., 1983. Grazing of intertidal algae by marine invertebrates. Oceanogr. Mar. Biol. Ann. Rev. 21, 195-282.

Kaehler, S., Williams, G.A., 1996. Distribution of algae on tropical rocky shores: spatial and temporal patterns of non-coralline encrusting algae in Hong Kong. Mar. Biol. $125,177-187$.

Little, C., 1989. Factors governing patterns of foraging activity in littoral marine herbivorous molluscs. J. Moll. Stud. 55, 273-284.
Little, C., Stirling, P., 1985. Patterns of foraging activity in the limpet Patella vulgata L. - a preliminary study. J. Exp. Mar. Biol. Ecol. 89, 283-296.

Liu, J.H., 1993. Activity rhythms and 'homing' behaviour by two pairs of high and lowzoned intertidal limpets in Hong Kong. In: Morton, B. (Ed.), The marine biology of the south China sea. Proceedings of the first international conference on the marine biology of Hong Kong and the south China Sea. Hong Kong University Press, pp. 471-492.

Morgan, S.G., 2007. Tidal rhythms. In: Denny, M.W., Gaines, S.D. (Eds.), The Encyclopaedia of tidepools and rocky shores. University of California Press, pp. 469-473.

Morton, B.S., Morton, J.E., 1983. The seashore ecology of Hong Kong. Hong Kong University Press, Hong Kong.

Morton, B.S., Williams, G.A., Lee, S.Y., 1996. The benthic marine ecology of Hong Kong: a dwindling heritage? Coastal infrastructure development in Hong Kong: A review, pp. 233-267.

Naylor, E., 1958. Tidal and diurnal rhythms of locomotory activity in Carcinus maenas (L.). J. Exp. Biol. 35, 602-610.

Naylor, E., 1988. Clock-controlled behaviour in intertidal animals. In: Chelazzi, G., Vannini, M. (Eds.), Behavioural adaptation to intertidal life. Plenum Press, pp. 1-14.

Naylor, E., 1989. Temporal aspects of adaptation in the behavioural physiology of marine animals. In: Klekowski, R.Z. (Ed.), 21st European Marine Biology Symposium, Gdansk. Polish Academy of Sciences, pp. 123-135.

Ng. J.S.S., Williams, G.A., 2006. Intraspecific variation in foraging behaviour: influence of shore height on temporal organization of activity in the chiton Acantholeura japonica. Mar. Ecol. Prog. Ser. 321, 183-192.

Ngan, A. 2006. Environmental stress and its implications for behavioural plasticity of foraging in Cellana grata. Unpublished Ph.D. thesis, The University of Hong Kong, Hong Kong.

Orton, J.H., 1929. Observations on Patella vulgata Part III: Habitat and habits. J. Mar. Biol Ass. UK 16, 277-288.

Palmer, J.D., 1995. The biological rhythms and clocks of intertidal animals. Oxford University Press.

Petpiroon, S., Morgan, E., 1983. Observations on the tidal activity rhythm of the periwinkle Littorina nigrolineata. (Gray). Mar. Behav. Phys. 9, 171-192.

Statgraphics, 1991. Statgraphics reference manual. Maugistics, Rockville, Maryland 20852, USA.

Williams, G.A., 1993. The relationship between herbivorous molluscs and algae on moderately exposed Hong Kong shores. In: Morton, B.S. (Ed.), The marine biology of the South China Sea. Proceedings of the first international conference on the marine biology of the South China Sea. Hong Kong University Press, Hong Kong, pp. 459-470.

Williams, G.A., 1994. The relationship between shade and molluscan grazing in structuring communities on a moderately exposed tropical rocky shore. J. Exp. Mar. Biol. Ecol. 178, 79-95.

Williams, G.A., Morritt, D., 1995. Habitat partitioning and thermal tolerance in a tropical limpet, Cellana grata. Mar. Ecol. Prog. Ser. 124, 89-103.

Williams, G.A., Little, C., 2007. Foraging behaviour. In: Denny, M.W., Gaines, S.D. (Eds.), The Encyclopaedia of tidepools and rocky shores. University of California Press, pp. 239-242

Zann, L.P., 1973. Relationships between intertidal zonation and circatidal rhythmicity in littoral marine gastropods. Mar. Biol. 18, 243-250. 